\title{
Preliminary Hearing Results of Tympanomastoidectomies Using Titanium Prostheses: Scenario in a Developing Country*
}

\author{
Ankush Sayal, Virangna Taneja, Achal Gulati\# \\ Department of Otolaryngology and Head \& Neck Surgery, MAM College and Assoc., LN Hospital, New Delhi, India \\ Email: \#achalgulati@rediff.com, ankushsayal@yahoo.com, virangnataneja@ymail.com
}

Received June 23, 2013; revised July 20, 2013; accepted August 8, 2013

Copyright (C 2013 Ankush Sayal et al. This is an open access article distributed under the Creative Commons Attribution License, which permits unrestricted use, distribution, and reproduction in any medium, provided the original work is properly cited.

\begin{abstract}
The study was done to review postoperative hearing gain of patients with different Middle Ear Risk Index (MERI) undergoing Tympanomastoidectomies with titanium prostheses reconstruction. A Retrospective chart review was performed from September 2009 to December 2011. Of the 17 cases, 9 had moderate MERI while 8 had severe MERI. 9 patients underwent Tympanomastoidectomy with Total Ossicular Reconstruction Prostheses (TORP) and 8 with Partial Ossicular Reconstruction Prostheses (PORP). Hearing gain of $25.31 \mathrm{~dB}$ was achieved in cases with moderate MERI compared to $29.37 \mathrm{~dB}$ in severe MERI. Less than $20 \mathrm{~dB}$ average air-bone gap was achieved in $75 \%$ of PORP and $77.77 \%$ of TORP reconstructions. In developing countries with limited resources, decision regarding ossicular reconstruction should be made taking into account MERI, intra operative findings and type of surgery. Best results are achieved in cases of CWD with TORP and ICW with PORP.
\end{abstract}

Keywords: Midddle Ear Risk Index; Titanium; Total Ossicular Replacement Prosthesis; Partial Ossicular Replacement Prosthesis

\section{Introduction}

The foremost objective of surgery done for chronic otitis media is complete exteriorisation of disease. Although the concept of disease removal was resolved much earlier, the problem of reconstruction and giving better listening to the patient have remained a challenge for the otologist. In early 1960, John Sheehy described the use of prosthesis for reconstruction of the ossicular chain and since then various prostheses have been used in the form of hydroxylapatite, Plastipore, Polyethylene and most recently titanium prosthesis. The titanium implant is compatible, readily available, light weight $(4 \mathrm{mg})$ and technically easy to use [1-2]. These titanium implants have overcome the problems associated with the use of autologus implants (usually necrosed due to underlying disease) and homograft's (risk of transmission of underlying disease), but their use is limited due to high costs.

In the past studies by Tos [3], Hirsch [4], Vartianer [5], they concluded that disease exteriorisation as well as

\footnotetext{
*Financial Disclosure: The study was conducted at MAM College and Assoc. LN Hospital, and the author and co-authors are employed at the same institution. There are no financial interests involved.

${ }^{\#}$ Corresponding author.
}

ossicular reconstruction should be done in the same setting. Contrary to this, studies by Sheehy [6] and Shelton [7] advocated the role of staged surgery to provide a better hearing outcome. They concluded that the 2nd stage surgery should be performed after a period of $6-24$ months to relook for any recurrence and for the purpose of ossicular reconstruction. But the concept of staging has since then remained a matter of controversy and the decision is best left to the operating surgeon who may decide depending on the intra operative findings and the chances of the patient returning for regular follow-up, a situation often encountered in the developing countries. In order to grade the severity of middle ear disease. Kartush developed a practical reporting protocol to stratify patient groups to allow for meaningful study comparisons. This numerical indicator has been referred to as "Middle Ear Risk Index".

To determine Middle Ear Risk Index (MERI) of a patient, a specific value is assigned for each risk factor, and then the value which includes Belluci criteria to assess the degree of otorrhoea, Austin/Kartush criteria for ossicular status, perforation, Cholesteatoma middle ear granulations/effusion and history of previous surgery [8] was 
added. The suggested risk categories can be derived from MERI as follows: MERI $0=$ Normal; MERI $1-3=$ Mild disease; MERI 4 - 6 = Moderate disease; MERI 7 - $12=$ Severe disease.

Being in a developing country with limited economic and human resources, an important objective of ear surgery is not only to provide good listening to the individual, but also provide the same at affordable costs. Paucity of resources and the fact that most patients in our country present with severe MERI, the use of such costly titanium prostheses becomes limited only to patients where maximum benefit is expected. Therefore in such a scenario planning, -ossicular, -reconstructions in any patient undergoing Tympanomastoidectomy requires precise judgement and population-based studies for the effective use of limited resources. But even after an extensive review of literature, we found that similar studies are lacking. This study compares the audiological improvement in patients with moderate/severe MERI undergoing Tympanomastoidectomies (Canal Wall Up or Canal Wall Down) and reconstruction using titanium TORP or PORP. We have used Spiggle and Theis titanium TORP and PORP in this study for the ossicular reconstruction. Available length for TORP varies from $7 \mathrm{~mm}$ to $3.5 \mathrm{~mm}$, compared to $3.5 \mathrm{~mm}$ to $0.5 \mathrm{~mm}$ for PORP.

\section{Materials and Methods}

A retrospective analysis was done for all the patients undergoing tympanomastoidectomy with ossicular chain reconstruction from September 2009 to December 2011 (28 months). Patients of chronic otitis media with Cholesteatoma or granulations with a purely conductive hearing loss were included in the study. Those with history of complications or have undergone previous ear surgeries were excluded from the study. Of the total, complete follow up with post operative audiological analysis was available for 17 cases.

Data was analysed in terms of middle ear risk index, surgical procedure being done (ICW or CWD), method of reconstruction (TORP or PORP), single/2nd stage reconstruction, and compared with the audiological improvement. Pre operative as well as post operative AirBone gap was calculated at 0.5, 1, 2 and $3 \mathrm{kHz}$ in accordance with the guidelines of American Academy of Otolaryngology_Head and Neck Surgery committee on hearing and equilibrium for hearing evaluation [9].

All the surgeries were performed under General Anaesthesia via Post aural approach by the same surgeon (A.S). The MERI index, disease extent and the condition of middle ear mucosa were used as criteria by the operating surgeon intra operatively for deciding whether to go for canal wall up or canal wall down surgery, and also for deciding whether the reconstruction would be done as a primary reconstruction or to subject the patient to a sec- ond stage reconstruction. Depending on the ossicular status, TORP or PORP were placed over the stapes footplate or supra structure respectively. The size of the prosthesis was calculated using a measuring gauge. For TORP, the length of the prostheses required was calculated from footplate of stapes while for PORP, the stapes head was used as a reference. The upper limit was taken till the facial ridge in case of CWD and till the annulus in case of ICW surgery for both the prostheses. A small piece of conchal cartilage ( $0.3 \mathrm{~mm}$ thickness) was harvested in all the cases and placed over the prostheses.

\section{Results}

Of the 17 cases that fulfilled the inclusion criteria, 9 had moderate MERI and 8 had severe MERI. 7 patients underwent mastoidectomies with canal wall down, while 10 had intact canal wall surgery. 8 underwent reconstruction using PORP, whereas 9 cases underwent reconstruction using TORP. 13 cases were taken up for disease removal and reconstruction in single setting, whereas 4 cases were taken up for 2nd stage reconstruction (Table 1).

6 cases underwent ICW with PORP reconstruction and 4 cases underwent ICW with TORP reconstruction. In 5 Canal wall down with TORP reconstruction was attempted and 2 cases underwent CWD with PORP reconstruction (Figure 1). Average hearing gain was found to be $28.75 \mathrm{~dB}$ in 10 cases undergoing ICW, compared to 7 cases undergoing CWD who had average postoperative hearing of $21.25 \mathrm{~dB}$. Postoperatively, the mean Air-Bone Gap was $17.5 \mathrm{~dB}$ for cases which underwent reconstruction using PORP (Figure 2) compared to $17.22 \mathrm{~dB}$ in which TORP (Figure 3) were used. Mean hearing gain in the PORP group was $26.87 \mathrm{~dB}$, whereas for those undergoing reconstruction using TORP was $28.89 \mathrm{~dB}$ (Table 2). $50 \%$ of patients undergoing reconstruction with PORP (4 out of 8 ) and 45\% with TORP (4 out of 9) had postoperative hearing between $10-20 \mathrm{~dB}$.

Of the 8 cases having severe MERI, 5 (62.5\%) required CWD surgery whereas 3 (37.5\%) required ICW. On the other hand, of the 9 cases having moderate MERI, only 2 (22.2\%) required CWD compared to 7 (77.8\%) who required ICW. All the 8 cases having severe MERI underwent reconstruction using TORP. On the other hand 9 cases had moderate MERI of which 8 underwent PORP insertion and 1 had TORP insertion. All 9 patients having moderate MERI underwent single stage reconstruction, whereas of the 8 cases having severe MERI, 4 underwent single stage and 4 underwent 2nd stage reconstruction. 8 cases having severe MERI had average post operative hearing gain of $29.37 \mathrm{~dB}$. On the other hand 9 cases having moderate MERI had average post operative hearing gain of $25.62 \mathrm{~dB}$ with PORP and hearing gain of $25 \mathrm{~dB}$ with TORP making an average gain of $25.31 \mathrm{~dB}$ (Tables 3 and 4). 
Table 1. Comparison of pre and post operative hearing status.

\begin{tabular}{|c|c|c|c|c|c|c|}
\hline S.no & Patient details & Surgery & $\begin{array}{l}\text { Preoperative hearing } \\
\text { A-B gap (dB) }\end{array}$ & $\begin{array}{c}\text { Postop hearing A-B } \\
\text { gap (dB) }\end{array}$ & Hearing gain $(\mathrm{dB})$ & MERI \\
\hline 1 & Md Nazim 19 yrs/M & ICW with PORP & 50 & 15 & 35 & Moderate \\
\hline 2 & Jagdish 19 yrs/M & CWD with PORP & 35 & 15 & 20 & Moderate \\
\hline 3 & Sanju devi 27 yrs/F & CWD with TORP & 50 & 35 & 15 & Severe \\
\hline 4 & Baleshwari 36 yrs/F & ICW with TORP & 35 & 20 & 15 & Severe \\
\hline 5 & Chandani 24 yrs/F & ICW with TORP & 50 & 15 & 35 & Severe \\
\hline 6 & Salman 14 yrs/M & ICW with PORP & 60 & 30 & 30 & Moderate \\
\hline 7 & Sunil 25 yrs/M & CWD with TORP & 45 & 25 & 20 & Severe \\
\hline 8 & Atiq rehman $17 \mathrm{yrs} / \mathrm{M}$ & ICW with PORP & 30 & 10 & 20 & Moderate \\
\hline 9 & Zahid 25 yrs/M & ICW with PORP & 50 & 15 & 35 & Moderate \\
\hline 10 & Manish 22 yrs/M & CWD with 2nd stage TORP & 50 & 15 & 35 & Severe \\
\hline 11 & Santosh 25 yrs/M & ICW with TORP & 40 & 15 & 25 & Moderate \\
\hline 12 & Nasir 18 yrs/M & ICW with PORP & 40 & 20 & 20 & Moderate \\
\hline 13 & Rani 19 yrs/F & CWD with 2nd stage TORP & 50 & 10 & 40 & Severe \\
\hline 14 & Rizwana 30 yrs/F & ICW with 2nd stage TORP & 45 & 10 & 35 & Severe \\
\hline 15 & Kavita 20 yrs/F & ICW with PORP & 50 & 10 & 40 & Moderate \\
\hline 16 & Shehzadi 16 yrs/F & CWD with 2nd stage TORP & 50 & 10 & 40 & Severe \\
\hline 17 & Kalpana 25 yrs/F & CWD with PORP & 30 & 25 & 5 & Moderate \\
\hline
\end{tabular}

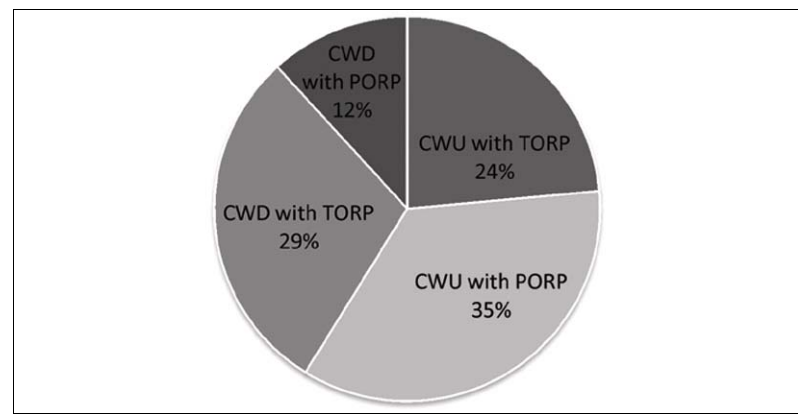

Figure 1. Type of surgery with reconstruction.

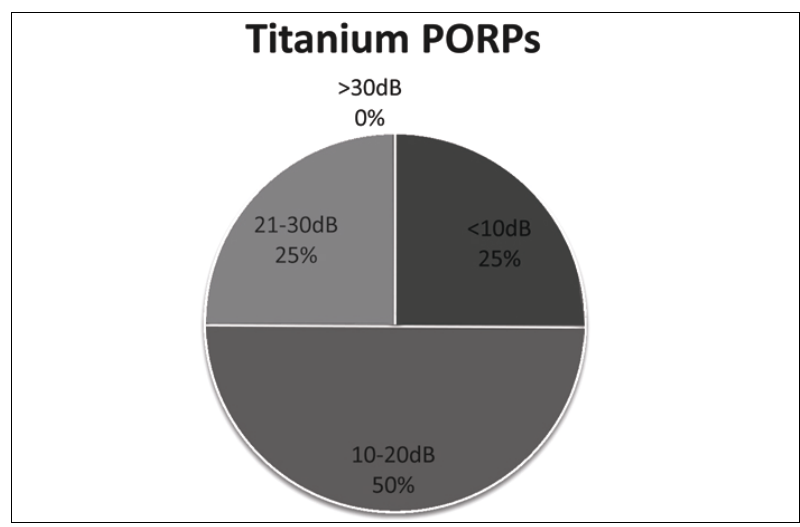

Figure 2. Audiological improvement using PORP.

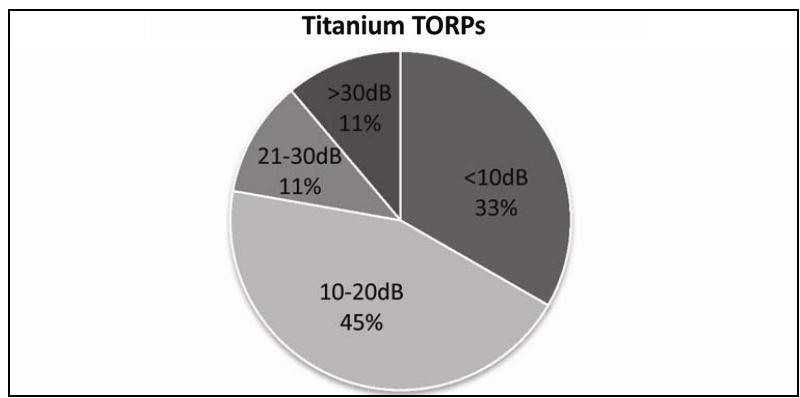

Figure 3. Audiological improvement using TORP.

We found that our results were best in ICW using PORP reconstruction and with CWD with TORP being $30 \mathrm{~dB}$ each. Hearing gain was found to be $27.5 \mathrm{~dB}$ in ICW with TORP and $12.5 \mathrm{~dB}$ in CWD with PORP (Figures 4-7).

Of all 17 cases 13 underwent Tymapanomastoidectomy and ossicular chain reconstruction in same sitting compared to 4 who underwent 2nd stage reconstruction and all 4 required CWD (Table 5). Average hearing gain of 13 patients undergoing single stage reconstruction was $19.58 \mathrm{~dB}$ compared to $30 \mathrm{~dB}$ for cases undergoing 2nd stage reconstruction. 3 patients required revision surgeries (patient number 5, 6 and 13). 2 patients who had TORP placement found no postoperative hearing gain 
Table 2. Average audiological hearing gain post Tympanomastidectomy with ossicular reconstruction.

\begin{tabular}{lccc}
\hline & $\begin{array}{c}\text { Average } \\
\text { Preoperative } \\
\text { hearing }(\mathrm{dB})\end{array}$ & $\begin{array}{c}\text { Average } \\
\text { Postoperative } \\
\text { hearing }(\mathrm{dB})\end{array}$ & $\begin{array}{c}\text { Average } \\
\text { Hearing } \\
\text { gain }(\mathrm{dB})\end{array}$ \\
\hline ICW with TORP & 42.5 & 20 & 27.5 \\
ICW with PORP & 46.66 & 16.66 & 30 \\
CWD with TORP & 49 & 19 & 30 \\
CWD with PORP & 32.5 & 20 & 12.5 \\
\hline
\end{tabular}

Table 3. Audiological hearing gain in terms of Middle Ear Risk Index.

\begin{tabular}{ccc}
\hline MERI & $\begin{array}{c}\text { Hearing gain in } \\
\text { patients with TORP }\end{array}$ & $\begin{array}{c}\text { Hearing gain in } \\
\text { patients with PORP }\end{array}$ \\
\hline Moderate & 25 & 25.625 \\
Severe & 29.37 & no case \\
\hline
\end{tabular}

Table 4. Average audiological hearing gain in terms of MERI and type of reconstruction.

\begin{tabular}{ccccc}
\hline MERI & $\begin{array}{c}\text { ICW with } \\
\text { PORP }\end{array}$ & $\begin{array}{c}\text { ICW with } \\
\text { TORP }\end{array}$ & $\begin{array}{c}\text { CWD with } \\
\text { PORP }\end{array}$ & $\begin{array}{c}\text { CWD with } \\
\text { TORP }\end{array}$ \\
\hline Moderate & $\begin{array}{c}30 \mathrm{~dB} \\
\text { (6 patients) }\end{array}$ & $\begin{array}{c}25 \mathrm{~dB} \\
(1 \text { patient) }\end{array}$ & $\begin{array}{c}12.5 \mathrm{~dB} \\
(2 \text { patients) }\end{array}$ & No patient \\
Severe & No patient & $\begin{array}{c}28.33 \mathrm{~dB} \\
\text { (3 patients) }\end{array}$ & No patient & $\begin{array}{c}\text { 30dB } \\
\text { (5 patients) }\end{array}$ \\
\hline
\end{tabular}

Table 5. Patient distribution in terms of staged reconstruction.

\begin{tabular}{ccc}
\hline MERI & Single stage reconstruction & 2nd stage reconstruction \\
\hline Moderate & 8 & 1 \\
Severe & 5 & 3 \\
\hline
\end{tabular}

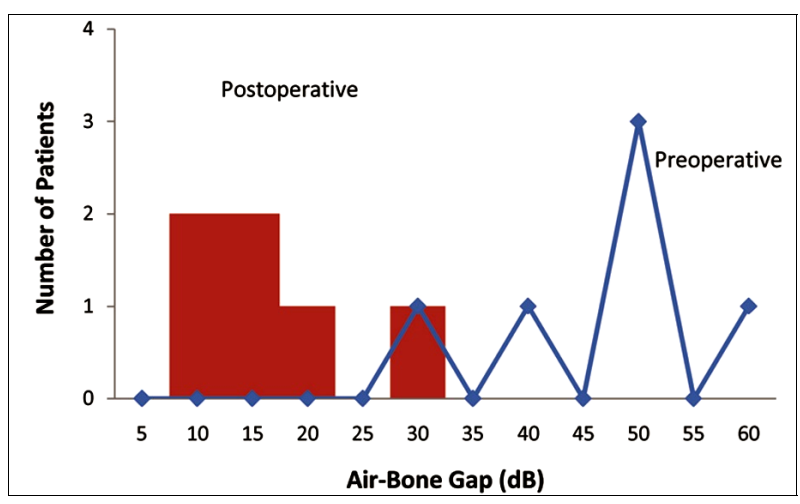

Figure 4. Hearing results of ICW with PORP.

and therefore exploration was done, whereas 1 patient with PORP insertion (intra operative-limited cholesteatoma confined to attic with necroses of incus and malleus

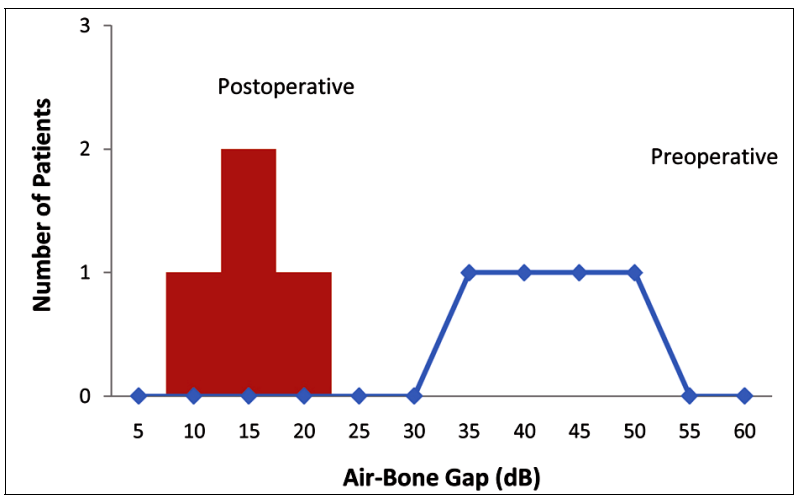

Figure 5. Hearing results of ICW with TORP.

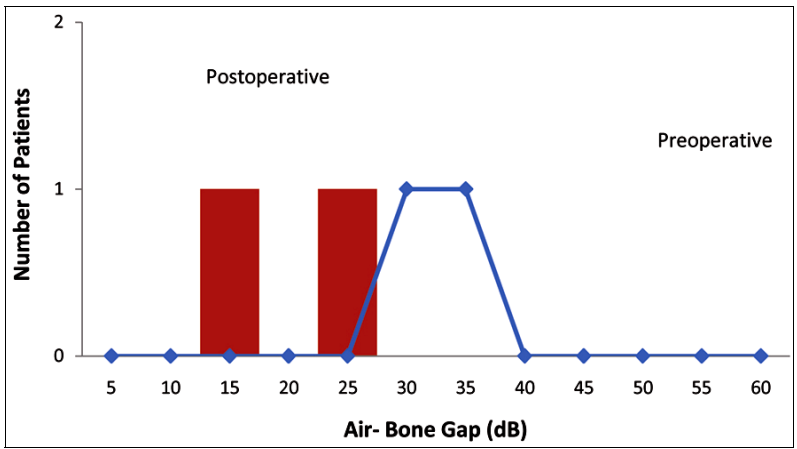

Figure 6. Hearing results of CWD with PORP.

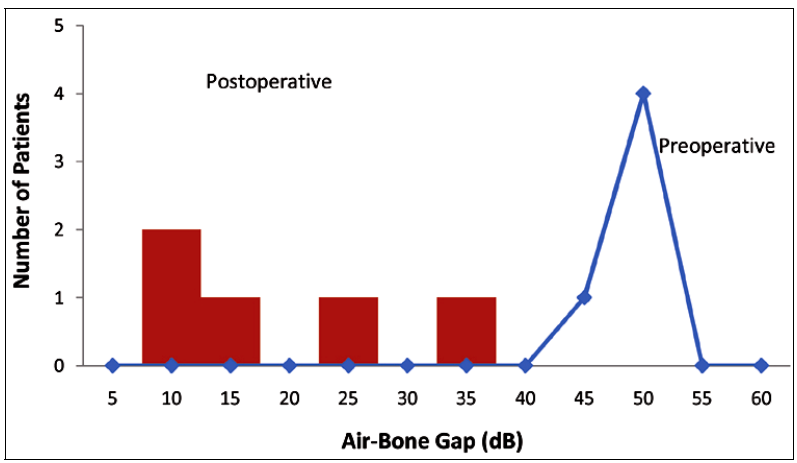

Figure 7. Hearing results of CWD with TORP.

but intact stapes supra structure) who initially had hearing gain later developed infection and had PORP extrusion, is still awaiting revision surgery (Figure 8). Follow up of cases was ranging from 4 month to 32 months (average 18 months).

\section{Discussion}

Of 8 cases having severe MERI, 5 (62.5\%) required CWD surgery and 3 (37.5\%) required ICW. All underwent reconstruction using TORP with an average post operative hearing gain of $29.37 \mathrm{~dB}$. On the other hand, of the 9 cases having moderate MERI, only 2 (22.2\%) required CWD while 7 (77.78\%) underwent ICW. 1 patient with CWD underwent TORP insertion with hearing gain 


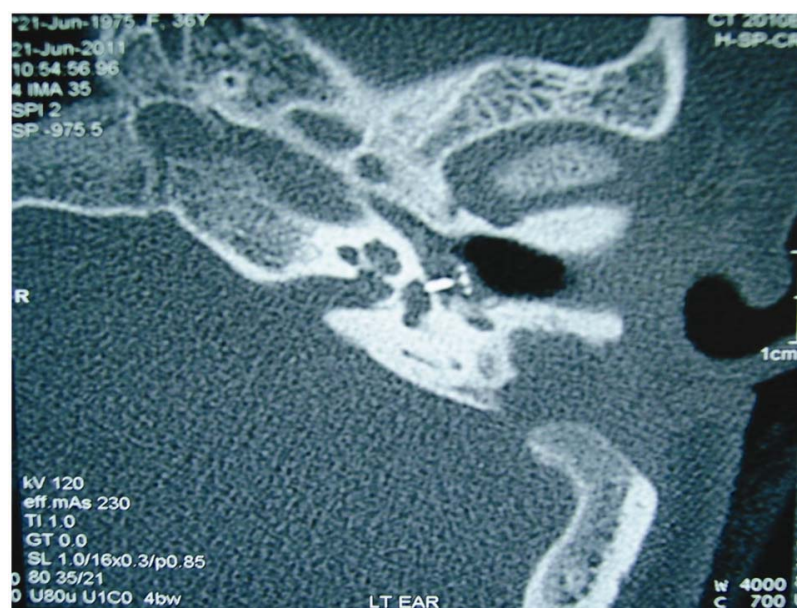

Figure 8. Postoperative CT scan of patient with TORP in situ (Lt Ear).

of $25 \mathrm{~dB}$ while 8 underwent PORP insertion with average post operative hearing gain of $25.62 \mathrm{~dB}$, making an average gain of $25.31 \mathrm{~dB}$. Cases of severe MERI with extensive disease usually required CWD approach with reconstruction using TORP. Therefore the selection of the type of prostheses is essential in a developing country with limited resources and resource allocation for procurement of TORP and PORP should be done in accordance with the type of patients (moderate or severe MERI) presented to hospital in such countries. But even after an extensive review of literature, we found that similar studies are lacking. This study was aimed to report the efficacy of titanium ossicular prostheses in developing country with limited resources where most patients of country have severe MERI at the time of presentation.

In our series of 17 patients, the average improvement of ABG was $27.88 \mathrm{~dB}$ (28.89 dB for TORP and 26.87 dB for PORP) at the end of follow up of 4 - 32 months (average being 18 months). Although hearing improvement was seen with both TORP and PORP, best results were seen with ICW with PORP placement and CWD with 2nd stage TORP placement i.e. an improved hearing gain of $30 \mathrm{~dB}$.

A large multicentre series conducted by Begall et al. [10] on 528 patients reported a hearing improvement of $15 \mathrm{~dB}$, especially at low frequencies. A study done by Stupp et al. [11,12] found a success rate of 76\%, studies by Ho et al. [13] and Gardner et al. [14] have reported success rate of 56\% (64\% for PORP and $45 \%$ for TORP) and $71 \%$ for PORP and $44 \%$ for TORP respectively. In the present study success was considered after attaining dry ear and with a postoperative improvement of an Air Bone Gap of $20 \mathrm{~dB}$ or less and our success rate was $76.47 \%$ (75\% for PORP and $77.77 \%$ for TORP).

In the current study, factors affecting the audiometric results included the type of surgical procedure (CWU or CWD), and presence of ossicular status intra operatively.
In the study, we found that the use of TORP with CWD/ ICW is associated with a poorer functional outcome when compared to PORP with CWD/ICW in terms of postoperative hearing gain $(90 \%$ of ICW \{with TORP and PORP $\}$ and 57.14\% of CWD \{with TORP and PORP had post operative hearing of less than $20 \mathrm{~dB}$ ). A study by Martin et al. [15] also reported that ICW procedure achieved a better hearing outcome with titanium prostheses than with CWD procedure.

In our study 3 patients required revision surgery, in which 2 (11.76\%) had no hearing improvement and 1 (5.88\%) had extrusion owing to middle ear infection. Extrusion rates in our study were comparable to those of Ho et al. [13] (4\%) and of Begall et al. [10] (4.4\%). Rates of revision surgery were higher compared to other studies by Stupp et al. [11] (5.6\%), Martin and Harner [15] (7\%) and Gardner et al. [14] (6\%).

Our functional results were better in cases that underwent Tympanomastoidectomy with 2nd stage ossicular reconstruction compared to those who underwent reconstruction in single sitting. The postoperative hearing gain was $19.58 \mathrm{~dB}$ after primary reconstruction of ossicular chain compared to $30 \mathrm{~dB}$ after 2nd stage reconstruction. Therefore in cases having severe MERI with ossicular destruction and Cholesteatoma it is advisable to stage the surgery.

\section{Conclusion}

We believe that Titanium ossicular implant is effective in achieving the goal of hearing improvement due to their light weight, inertness and tissue compatibility but in view of their high costs with availability of limited resources, their use should be decided judiciously. Best results are achieved when using CWD with TORP and ICW with PORP. We also conclude that in severe MERI, it is best to stage the surgery.

\section{Summary}

Postoperative hearing gains of patients with different Middle Ear Risk Index (MERI) who underwent Tympanomastoidectomies and reconstruction using titanium prostheses was done over a span of 27-months. The results were analyzed according to guidelines of American Academy of Otolaryngology-Head and Neck Surgery. Hearing gain of $25.31 \mathrm{~dB}$ was achieved in cases with moderate MERI whereas hearing gain of $29.37 \mathrm{~dB}$ was achieved in cases with severe MERI. Average Postoperative pure-tone air-bone gap was found to be $17.22 \mathrm{~dB}$ in total ossicular reconstruction compared to $17.50 \mathrm{~dB}$ of partial ossicular reconstruction. Less than $20 \mathrm{~dB}$ pure tone average air-bone gap was achieved in $75 \%$ of partial ossicular chain reconstructions and $77.77 \%$ of total ossicular chain reconstructions. In developing countries 
with limited resources, decision regarding ossicular reconstruction should be made taking into account MERI, intra operative findings and type of surgery to achieve better post operative hearing results. Best results are achieved in cases of CWD with TORP and ICW with PORP.

\section{REFERENCES}

[1] L. M. Menendez-Colino, M. Bernal-Sprekelsen, I. Alobid and J. Traserra-Coderch, "Preliminary Functional Results of Tympanoplasty with Titanium Prostheses," Otolaryngology-Head and Neck Surgery, Vol. 131, No. 5, 2004, pp. 747-749. doi:10.1016/j.otohns.2004.04.017

[2] C. V. Dalchow, D. Grun and H. F. Stupp, "Reconstruction of the Ossicular Chain with Titanium Implants," Otolaryngology-Head and Neck Surgery, Vol. 125, No. 6, 2001, pp. 628-630. doi:10.1067/mhn.2001.120397

[3] M. Tos, "Late Results in Tympanoplasty," Acta Oto-Laryngologica, Vol. 82, No. 1, 1976, pp. 282-285. doi:10.3109/00016487609120906

[4] B. E. Hirsch, D. B. Kamerer and S. Doshi, "Single Stage Management of Cholesteatoma,” Otolaryngology_Head and Neck Surgery, Vol. 106, 1992, pp. 351-354.

[5] E. Vartianen and J. Nuutinen, "Long Term Hearing Results of One Stage Tympanoplasty for Chronic Otitis Media," European Archives of Oto-Rhino-Laryngology, Vol. 249, No. 6, 1992, pp. 329-331.

[6] J. L. Sheehy and J. A. Crabtree, "Tympanoplasty: Staging the Operation,” Laryngoscope, Vol. 83, No. 10, 1973, pp. 1594-1621. doi:10.1288/00005537-197310000-00003

[7] C. Shelton and J. L. Sheehy, "Tympanoplasty: Review of 400 Staged Cases,” Laryngoscope, Vol. 100, No. 7, 1990, pp. 679-681.
[8] J. M. Kartush, "Ossicular Chain Reconstruction. Capitulum to Malleus," Otolaryngologic Clinics of North America, Vol. 27, No. 4, 1994, pp. 689-715.

[9] American Academy of Otolaryngology-Head and Neck Surgery Foundation, Inc., "Committee on Hearing and Equilibrium Guidelines for the Evaluation of Results of Treatment of Conductive Hearing Loss,” Otolaryngology -Head and Neck Surgery, Vol. 113, No. 3, 1995, pp. 186-187. doi:10.1016/S0194-5998(95)70103-6

[10] K. Begall and H. Zimmermann, "Reconstruction of the Ossicular Chain with Titanium Implants. Results of a Multicenter Study,” Laryngorhinootologie, Vol. 79, No. 3, 2000, pp. 139-145. doi:10.1055/s-2000-298

[11] C. H. Stupp, C. Dalchow, D. Grun, H. F. Stupp and J. Wustrow, "Three Years of Experience with Titanium Implants in the Middle Ear," Laryngorhinootologie, Vol. 78, No. 6, 1999, pp. 299-303. doi:10.1055/s-2007-996875

[12] C. H. Stupp, H. F. Stupp and D. Grun, "Replacement of Ear Ossicles with Titanium Prostheses," Laryngorhinootologie, Vol. 75, No. 6, 1996, pp. 335-337. doi:10.1055/s-2007-997590

[13] S. Y. Ho, R. A. Battista and R. J. Wiet, "Early Results with Titanium Ossicular Implants,” Otology \& Neurotology, Vol. 24, No. 2, 2003, pp. 149-152. doi:10.1097/00129492-200303000-00005

[14] E. K. Gardner, C. G. Jackson and D. M. Kaylie, "Results with Titanium Ossicular Reconstruction Prostheses," Laryngoscope, Vol. 114, No. 1, 2004, pp. 65-70. doi:10.1097/00005537-200401000-00011

[15] A. D. Martin and S. G. Harner, "Ossicular Reconstruction with Titanium Prostheses,” Laryngoscope, Vol. 114, No. 1, 2004, pp. 61-64. doi:10.1097/00005537-200401000-00010 